\title{
A fitness landscape approach to technological complexity, modularity, and vertical disintegration
}

\author{
Koen Frenken \\ Urban and Regional Research Centre Utrecht (URU), Faculty of Geosciences, Utrecht University, \\ P.O. Box 80115, NL-3508 TC, Utrecht, The Netherlands
}

Received September 2002; received in revised form September 2005; accepted January 2006

Available online 29 March 2006

\begin{abstract}
The biological evolution of complex organisms, in which the functioning of genes is interdependent, has been analysed as "hill-climbing" on NK fitness landscapes through random mutation and natural selection [Kauffman, S.A., 1993. The Origins of Order. Self-organization and Selection in Evolution. Oxford University Press, New York \& Oxford]. In evolutionary economics, NK fitness landscapes have been used to simulate the design of complex technological systems as a trial-and-error process towards local optima. Interdependencies among a system's elements do not only render the optimisation of product design a difficult task, but also put constraints on the vertical disintegration of production. In this context, evolutionary economists introduced the concept of "dynamic transaction costs" [Langlois, R.N., 1992. Transaction-cost economics in real time. Industrial and Corporate Change 1 (1), 99-127; Langlois, R.N., 2001. The vanishing hand: the changing dynamics of industrial capitalism, Paper presented at the workshop Reappraising Production Theory: Concepts, Cases and Models, Max Planck Institute, Jena, November 29-December 1]. A system integrator outsourcing the production of elements to supplier firms not only transfers production, but to a large extent looses competence and control to bring about innovations in these elements. This is problematic when the element that is being outsourced is functionally interdependent with other elements in the system. The main aim of the paper is to theorise about modular product architectures that allow for outsourcing while reducing the constraints on possible innovation. The primary way to achieve modular architectures is to introduce elements that function as standards mediating interdependencies between other elements. It is argued that only a generalised version of the NK-model, which is developed in this paper, is able to represent this specific quality of standards that render architectures modular. It is also explained how the model of modular architectures differs from the model of decomposable architectures developed in a previous study [Frenken, K., Marengo, L., Valente, M., 1999. Interdependencies, near-decomposability and adaptation. In: Brenner, T. (Ed.), Computational Techniques for Modelling Learning in Economics. Kluwer, Boston, pp. 209-244]. A number of implications are derived from the generalised NK-model model
\end{abstract}

E-mail address: k.frenken@geo.uu.nl.

URL: http://econ.geo.uu.nl/frenken/frenken.html. 
that can be taken up in empirical studies on patterns of vertical disintegration in the life-cycle evolution of specific products. An evolutionary framework of vertical integration is needed to supplement the theory of the product life-cycle.

(C) 2006 Elsevier B.V. All rights reserved.

JEL classification: B52; D23; D83; L22; L23; 032

Keywords: NK-model; Modularity; Decomposability; Vertical integration; Transaction costs economics

\section{Introduction}

A recent development in evolutionary economics has been the use of rugged fitness landscapes to model the space in which economic agents search for new technological or organisational designs. A particular useful model is the NK-model that represents a system by its dimensions and their interdependencies (Kauffman, 1993). This model has originally been developed as a model of biological evolution, but its formal structure allows for applications in the domain of the design of technologies and organisations (Auerswald et al., 2000; Fleming, 2001; Fleming and Sorenson, 2001; Frenken, 2006; Frenken et al., 1999; Frenken and Nuvolari, 2004; Kauffman et al., 2000; Kauffman and Macready, 1995; Levinthal, 1997; Levinthal and Warglien, 1999; Marengo et al., 2000; McKelvey, 1999; Rivkin, 2000, 2001; Rivkin and Siggelkow, 2003; Siggelkow and Levinthal, 2003; Valente, 2000).

In a more generalised NK-model it is possible to simulate a fitness landscape in which the number of the system elements does not necessarily equal the number of the functions a system delivers for its users (Altenberg, 1995, 1997; Frenken, 2006). Using this model, one can understand technical standards as elements in a system, which do not affect particular functions, but solely mediate the interdependencies among other elements. Below, I will use Altenberg's generalised framework to theorise about outsourcing and modularity in complex technological systems. Two main arguments are derived that run as follows.

First, short-run profit can be maximised by outsourcing all elements of a complex system that can be acquired at lower prices (including transaction costs) through market relations. However, long-run profit also depends on a firm's ability to achieve higher product quality or lower prices through product or process innovation. Since innovation in complex systems requires coherent changes in interdependent elements of the system, coordination is required in the R\&D phase. To the extent that the competence to produce an element is related to the competence to innovate in this element, outsourcing the production of elements of a complex system entails the risk of losing the competence to innovate (Winter, 1988). The long-term competitiveness of a firm can be harmed when short-term profit is sought through outsourcing. It is argued that the generalised NK-model as an evolutionary model explains outsourcing not only from a transaction-cost point of view, but also with reference to the dynamic implications for future innovation possibilities.

Second, given the trade-off between minimising the transaction costs in the short run and maximising future innovation possibilities, a strategic question holds what type of architectures allow for outsourcing of production without hampering future innovation. In this context, the generalised NK-model can distinguish between decomposable and modular systems. In decomposable systems innovation is not hampered at all, since interdependencies are absent between subsystems. In modular yet non-decomposable systems, innovation is possible in modules to the extent that the innovation meets the requirements of interface standards that integrate the modules into a system. As long as the integrator firm controls the interface standards, mod- 
ularity allows the integrator to outsource production without frustrating future innovation by suppliers.

A formal approach to innovation in complex systems based on the NK-model also provides one with the possibility to develop new metrics for empirical research. In particular, a new indicator of modularity is proposed that is based on the architecture of complex systems, and which can be used in empirical research. For example, using this indicator, one can start to test systematically whether modularity indeed triggers vertical disintegration, and also, whether these patterns evolve over time according to the product and industry life-cycle theories (Klepper, 1997).

The paper will discuss the generalised NK-model of complex systems (Section 2) and the way it can be used to theorise about outsourcing and innovation (Section 3). Finally, a modularity indicator is derived that can be used in empirical research into the degree of modularity of different designs (Section 4). I end with conclusions (Section 5).

\section{The NK-model of complex technological systems}

Complex systems are systems containing elements that are interrelated within a particular structure (Simon, 1969). The evolutionary properties of complex systems have been subject of research in theoretical biology (Kauffman, 1993). In biological organisms, interdependencies among genes imply a complex relation between an organism's genotype and an organism's phenotype. At the level of the genotype mutations occur that introduce new variants in a population. At the level of the phenotype, which is the ensemble of traits that make up an organism's fitness, natural selection operates in terms of differential rates of reproduction. Complexity in an organism means here that a mutation in one gene may not only change the functional contribution of the mutated gene to the entire phenotype, but can also affect the functional contributions of interrelated genes to the phenotype. For this reason, the existent set of genes put structural constraints on the possible directions in further evolution: a certain phenotypic trait can only be improved by a particular mutation when the improvement in one trait outweighs the negative by-effects of this mutation with respect to other traits. In biology, this insight has led scholars to conclude that natural selection cannot be expected to lead to perfectly adapted organisms (Kauffman, 1993).

\subsection{Design space}

Technological systems have also been described as complex systems, which are made up of elements that collectively attain one or a number of goals (Simon, 1969). The complexity in designing a technological system is due to the interdependence in the working of elements that make up the system. Therefore, the set of optimal choices for the elements with regard to elementspecific output variables may prove sub-optimal when the effects of interdependencies between elements are taken into account. The ensemble of elements has to be evaluated at the system level to assess the effect of each single element on the system as a whole. The need to evaluate the working of elements at the system level poses a problem of complexity. Since the number of possible combinations between different variants of elements is an exponential function of the number of elements, time resources put constraints on global optimisation (Simon, 1969).

Complex (technological) systems have been described analytically using the NK-model. The model describes a system by a string of $N$ dimensions $(n=1, \ldots, N)$ referred to as elements in a system. In the context of complex products, say motorised technologies, such elements typically include the type of engine, the type of material, the type of cooling device, etc. (Frenken, 2006; Kauffman, 1993). For each element $n$, there exist a number of states called "alleles". The alleles 
refer to possible variants of an element among which a firm can choose and are labelled by integers "0", "1", "2", "3". The number of alleles of element $n$ is described as $A_{n}$. Each design, then, can be coded as a string $s$ of length $N$ is described by the alleles $s_{1} s_{2} \ldots s_{N}$ and is part of a possibility set $S$, for which holds:

$$
s \in S ; \quad s=s_{1} s_{2} \ldots s_{N} ; \quad s_{n} \in\left\{0,1, \ldots, A_{n}-1\right\}
$$

The $N$-dimensional possibility space $S$ is called the "design space" of a system, and it includes all possible combinations between the alleles of elements (Bradshaw, 1992; Dennett, 1995). The size of the design space $S$ is given by:

$$
S=\prod_{n=1}^{N} A_{n}
$$

The combinatorial nature of a design space implies that its size increases exponentially for linear increases in $N$. As most technologies are high-dimensional ( $N$ is large) and can be constructed in many different ways ( $A$ is large), design spaces are much vaster than firms can effectively explore. The computational complexity of designing a complex system can be illustrated by the design space of early aircraft at the time of the Wright Brothers, which has been estimated to contain nine dimensions from which almost 13,000,000 designs could be constructed by combination of alleles (Bradshaw, 1992).

\subsection{Kauffman's original NK-model}

The interdependencies between elements in a complex system are called "epistatic relations". An epistatic relation from one element to another element implies that when the allele of the one element changes, this change affects both the functioning of the one element itself and the functioning of the element that it epistatically affects. Following the example of aircraft, when the type of engine affects the functionality of a type of wings, one speaks of an epistatic relation from the engine element to the wing element. Note that epistatic relations can work both ways (when the choice of wings would affect the functionality of the engine), but not necessarily so.

The ensemble of epistatic relations within a technological system is called a technology's internal structure (Saviotti, 1996; Simon, 1969) or architecture (Henderson and Clark, 1990). In the original NK-model (Kauffman, 1993) the analysis has been restricted to particular types of architectures that can be expressed by a parameter $K$, which stands for the number of elements that affect the functioning of each element. For example, the class of systems for which holds $K=1$ refers to systems with an architecture in which the functionality of each element depends on the choice of allele of the element itself and on the choice of the allele of one other element. This parameter can be considered an indicator of a system's complexity with $K=0$ being the least complex and $K=N-1$ the most complex architecture. Consider as an explanatory example, a system for which holds $N=3$ and $K=1$ with an architecture as specified in the square matrix in Fig. 1. The architecture specifies the following epistatic relations between three elements and their fitness $w_{n}$. By definition, architectures in Kauffman's NK-model are such that each element has an "own" function that changes when the element in mutated (as indicated by the X-es along the diagonal). Depending on the $K$-value of a system, other possible interactions are either present (x) or absent (-). The fitness value of the first element changes only if the element itself or the second element is mutated. The fitness value of the second element changes only if the element itself or the first element is mutated. And, the fitness value of the third element changes only if element itself or the first element is mutated. 


$\begin{array}{lllc} & \mathrm{n}=1 & \mathrm{n}=2 & \mathrm{n}=3 \\ \mathrm{w}_{1} & \mathbf{x} & \mathrm{x} & - \\ \mathrm{w}_{2} & \mathrm{x} & \mathrm{x} & - \\ \mathrm{w}_{3} & \mathrm{x} & - & \mathrm{x}\end{array}$

Fig. 1. Example of an architecture of $N=3$-system with $K=1$.

$\begin{array}{ccccc} & \mathrm{w}_{1} & \mathrm{w}_{2} & \mathrm{w}_{3} & \mathrm{~W} \\ 000: & 0.7 & 0.2 & 0.3 & 0.40 \\ 001: & 0.7 & 0.2 & 0.6 & 0.50 \\ 010: & 0.9 & 0.3 & 0.3 & 0.50 \\ 011: & 0.9 & 0.3 & 0.6 & 0.60 \\ \text { 100: } & 0.8 & 0.7 & 0.1 & 0.53 \\ \text { 101: } & 0.8 & 0.7 & 0.4 & 0.63 \\ \text { 110: } & 0.3 & 0.5 & 0.1 & 0.30 \\ \text { 111: } & 0.3 & 0.5 & 0.4 & 0.40\end{array}$

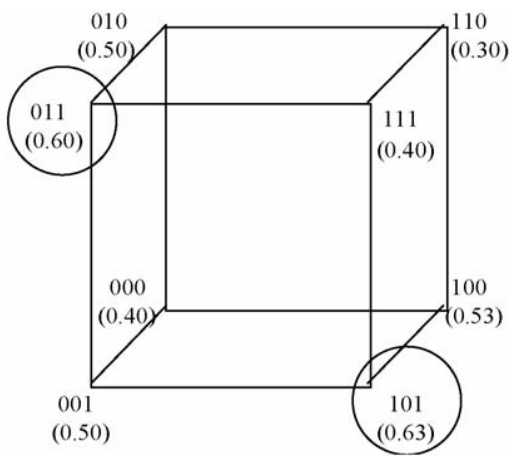

Fig. 2. Simulation of a fitness landscape of a $N=3$-system with $K=1$.

A fitness landscape is constructed by drawing randomly the value of the fitness of an element $w_{n}$ from the uniform distribution between 0 and 1 , each time it is itself mutated and each time an element with which it is epistatically related is mutated. System fitness, which can be considered product quality in the context of complex products, is derived as the mean value of the fitness of elements $^{1}$ :

$$
W(s)=\frac{1}{N} \sum_{n=1}^{N} w_{n}\left(s_{n}\right)
$$

A simulation of a fitness landscape is given in Fig. 2 that shows that fitness landscapes of complex systems with epistatic relations between elements can be characterised by the existence of multiple optima. Generally, one speaks of "rugged" fitness landscapes containing several peaks. Hillclimbing involves series of random mutations in one element. Each time a mutation leads to a string with higher fitness, the next mutation starts from this new string. This means that hillclimbing can end up in several optima. Search is "path-dependent" on the initial starting string and the sequence of mutations that follows. An example of path-dependence in the simulation example is when search starts from string 000 and the first random mutation leads to string 010, search will end up in the local optimum 011 . When search again starts from 000 and the first random mutation leads to 100 , search will end up in the global optimum 101. Note that path-dependence in the NK-model is different from the concept of path-dependence in models of increasing returns to adoption (Arthur, 1989; David, 1985). ${ }^{2}$

\footnotetext{
${ }^{1}$ Other functions are possible, but do not alter the concept of a fitness landscape as a mapping from a combinatorial design space onto a continuous fitness space (Frenken, 2001).

2 Path-dependence in the NK-model refers to the path of an individual designer hill-climbing a fitness landscape, which is dependent on the stochastic sequence of mutations in an otherwise unchanged fitness landscape. In models of network
} 
Contrary to biological evolution, human search can escape a local optimum by mutating several elements at the time. In the example, the sub-optimal string 011 can be left by mutating both the first and second elements at the same time thus "jumping" to the global optimum 101. Put another way, human search can always find the global optimum in principle by simply evaluating the fitness of all $S$ designs in design space. However, in large systems such an exhaustive search strategy is very expensive since the size of the design space increases exponentially with $N$ in Eq. (2). Exhaustive search quickly becomes inefficient as the search costs no longer outweigh the improvement in fitness of a global optimum over a local optimum (Frenken et al., 1999; Kauffman et al., 2000). This result provides the theoretical rationale for the use of the assumption of local search in evolutionary models.

\subsection{Altenberg's generalised NK-model}

The NK-model is based on the idea that each element in a system performs an "own" subfunction within the system with regard to the attainment of one overall function on which selection operates such as the relative capacity of each protein to catalyse a specific reaction (Kauffman, 1993). Each element $n$ is conceived to have a particular fitness value $w_{n}$ that reflects its functional contribution to the system as a whole. The fitness of the system as a whole is derived as the average of the fitness of individual elements. However, there is no reason to assume that systems contain as much functions as elements in the system.

A generalised NK-model can provide a general description of complex systems containing any number of elements and any number of functions as has been shown in a biological context (Altenberg, 1995, 1997). In biological systems, for which this generalised NK-model was conceived, an organism's $N$ genes are the system's elements and an organism's $F$ traits are the selection criteria. The string of genes constitutes an organism's genotype and the set of traits constitutes an organism's phenotype. The genotype of an organism is the level at which mutations take place, which are transmitted in its offspring. The phenotype is the level at which natural selection operates in terms of its relative fitness to the environment. A single gene may affect one or several traits in the phenotype and a single trait may be affected by one or several genes in the genotype. The number of traits that is affected by a particular gene in the genotype is referred to as a gene's "pleiotropy". The number of genes that affects a trait in the phenotype is referred to as a trait's "polygeny". The structure of epistatic relations between genes and traits is represented in a "genotype-phenotype map".

Analogously, a technological system can be described in terms of its $N$ elements and the $F$ functions it performs. The string of alleles of elements describes the "genotype" of a technological system, and the list of functions describes the "phenotype" of this system. Typical functions are speed, weight, comfort, safety, etc. The distinction between elements and functions is similar to the description of technology by a set of technical characteristics that are the object of design and service characteristics on which users select (Saviotti and Metcalfe, 1984; Saviotti, 1996).

A generalised model of complex technological systems will be used here as a model of technological design (Frenken and Nuvolari, 2004; Frenken, 2006). The architecture of a technology

externalities path-dependence refers to the sequence of agents adopting a technological option. As each new adopter increases the fitness value of a technology, a sequential adoption process can lead all agents to opt for one technology (lock-in). The former type of path-dependence can be called microscopic path-dependence, while the latter type of pathdependence can be called macroscopic path-dependence (Frenken, 2006). 


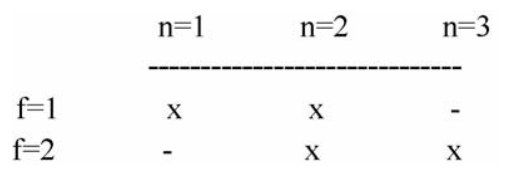

Fig. 3. Example of a generalised element-function matrix $(N=3, F=2)$.

is represented by an "element-function matrix" of size FxN with:

$$
M=\left[m_{f n}\right], \quad f=1, \ldots, F, \quad n=1, \ldots, N
$$

As in the NK-model, an epistatic relation is represented by $x$ when function $f$ is affected by element $n$ and by - when function $f$ is not affected by the element $n$. The original NK-model can now be understood as a special case of the generalised element-function matrices (Altenberg, 1997). For NK-systems, it holds that the number of functions $F$ equals the number of elements $N$. The NK-model also implies that the diagonal in the element-function matrix is always characterised by presence of a relation between element and function. Furthermore, the $K$-value in the NK-model implies that each function is affected by the same number of elements. Thus, in the NK-model the polygeny of each function is assumed to be equal to $K$, with pleiotropy of each element being determined randomly (with pleiotropy being on average equal to $K$ ). Dropping these two restrictions provides a general model of complex systems. An example of an element-function matrix of the generalised NK-model is given Fig. 3.

The way in which fitness landscape are constructed for generalised element-function matrices follows the same logic as the original NK-model discussed in the previous section. For each element that is mutated, all functions that are affected by this element are assigned a new, randomly drawn value from the uniform distribution between 0 and 1 . Total fitness is again derived as the mean of the fitness values of all functions:

$$
W(s)=\frac{1}{F} \sum_{f=1}^{F} w_{f}(s)
$$

A simulation of the fitness landscape example of the element-function matrix given in Fig. 3 is given in Fig. 4 for all possible combinations between two alleles of three elements. In this example, the first and third element each affect one function, but the second element affects both

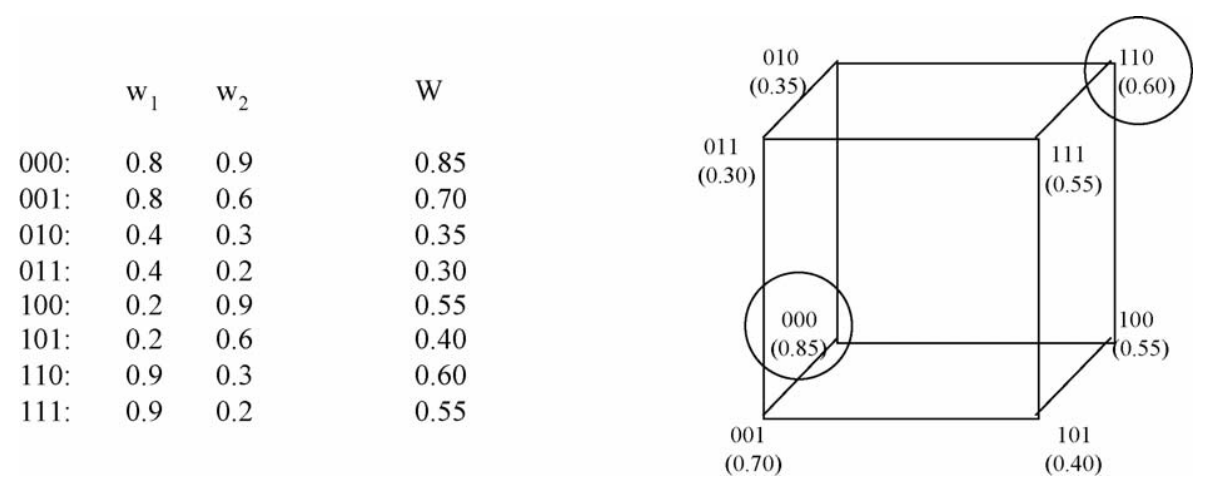

Fig. 4. Simulation of fitness landscape of the matrix in Fig. 3. 
functions. As long as the allele of the second element is fixed (either 0 or 1), the first element can be mutated in an attempt to raise the fitness value of the first function without affecting the second function. Similarly, the third element can be mutated to improve the fitness of the second function without affecting the first function. Once a choice is made for the allele of the second element (either 0 or 1), the other elements can be mutated until a local optimum is found ( 000 and 110 in this example). As explained below in more detail, the second element can be considered as a technical standard mediating the epistatic interaction between the other two elements, without having a particular function of its own.

\section{Decomposability, modularity and vertical disintegration}

\subsection{Static transaction costs}

Vertical disintegration is generally discussed with the framework of transaction costs (Williamson, 1975, 1979, 1985). Outsourcing of a particular intermediate good or service can be considered to be dependent on a comparison between cost of in-house production versus the cost of outsourcing (market price and transaction costs). Within an NK modelling framework, one can expect the complexity of each element in a complex technological system to bear on the transaction costs involved in outsourcing this element. More specifically, the transactions associated with outsourcing an element are expected to be a function of the element's pleiotropy (the number of epistatic relations from this element to other elements). The higher the number of interdependencies stemming from this element, the higher is the required accuracy of production. A small deviation of the desired properties of an element can create malfunctionings in possibly all other epistatically related elements (Williamson, 1979). Consequently, market transaction will involve higher transaction costs in particular in the writing and enforcement of contracts.

Following the example of the simulation in Figs. 3 and 4, one can expect, ceteris paribus, that transaction costs of outsourcing the second element are higher than that of outsourcing the first element or outsourcing the third element. The reason is that the second element is epistatically interdependent with two elements, while the first element and the third element both are epistatically related with only one element. This property holds independently of the specific design $s_{1} s_{2} s_{3}$ under consideration.

\subsection{Dynamic transaction costs}

The relation between pleiotropy and transaction costs, however, deals solely with the short-term static considerations of production costs of a given design. It does not take into account the longterm dynamic consequences of outsourcing elements regarding future possibilities of innovations that can be carried out either by the integrator firm or the supplier firm (or in collaboration). The existence of local optima with respect to one-element mutations implies that successful innovation generally requires changes in multiple elements at the same time. This analytical insight is consonant with the concept of "technological imbalances" (Rosenberg, 1969): successful innovation requires a series of coordinated mutations in several elements to achieve a new local optimum with higher fitness.

To find a new local optimum from an existing local optimum requires "long jumps" involving mutations in at least two elements. When the finding of a new local optimum would require mutations in both an element currently produced by the system integrator (or supplier firm 1) and an element currently produced by a supplier (or supplier 2), coordination is required. Future 
possibilities of innovation in different elements are extremely difficult to encode ex ante in market contracts. This difficulty stems for the inherently uncertain outcome of search activity on rugged landscapes due to epistasis between elements. Integrator and supplier cannot foreseen in which elements and at what moment mutations will prove successful in the future.

Coordination problems do not only arise when multiple firms aim to improve a complex product design. In the same vein coordination is required with respect to process innovation aimed at reducing costs of production. Again, the interdependencies among elements in a complex product designs generally involve interdependencies in the production system that is used to produce elements and assemble these into a product. Changes in particular stages of production require adaptation of related stages of production (Langlois, 1992, 2001; Buenstorf, 2005).

Though our perspective is focused on the coordination problem of the integrator firm, the coordination of innovation in complex systems is also problematic for the supplier of one or more elements. In pursuing $R \& D$ activity to improve the product quality or reduce production costs of the elements it offers, a supplier is bound to create malfunctioning in elements produced by the integrator firm itself or produced by other suppliers. Since a supplier firm often produces elements for several system integrators (by which scale economies can be realised that often explain outsourcing in the first place), the coordination problem becomes even harder, as a mutation in an element generally does not match with the different designs of different clients in which the elements must be integrated.

The above does not automatically imply that innovation in complex systems in which the production of elements are outsourced, always requires inter-firm collaborations. The system integrator can take on the role of sole innovator by exploring the complete design space on its own by means of in-house R\&D in all elements of the complex system. However, one can expect the competence of producing an element to be positively related to the competence of innovation in this dimension. In a complex systems framework, the concept of competence thus adds to transaction-cost theory, but in a dynamic sense. Outsourcing the production of an element in a complex system involves a loss in production competence with regard to this element. In as far as production competence relates to innovation competence, i.e. the competence to explore a dimension by means of mutation, integrator firms loose control over the future evolution of the complex system as a whole. One expects this innovation competence to decay with time, which suggests that temporary outsourcing of an element may still allow a firm to explore this dimension in a successful way, but that the probability of success will eventually decay over time.

Outsourcing the production of elements may thus decrease the rate of innovation of an integrator firm due to the loss of competence coherence, a conclusion that had been reached already on theoretical grounds (Foss, 1993; Langlois, 1992, 2001; Langlois and Foss, 1999; Mahnke, 2001; Williamson, 1999; Winter, 1988). Coherence in the NK-framework means a collection of competences to produce and innovate a set of epistatically related elements. In this context, the concept of dynamic transaction costs was introduced (Langlois, 1992; Teece, 1976) that arise when there exists 'a high degree of interdependence of successive stages of production'. The need to coordinate innovations in different elements of a complex system suggests that production may well be vertically integrated even when static transaction costs would suggest a vertically disintegrated organisation of production.

At the same time, outsourcing elements of a complex system leaves the integrator with a smaller design space allowing for more focus in R\&D and strategies (Prahalad and Hamel, 1990). The latter may bear a positive effect on the success of R\&D activity as the search space is reduced, but only to the extent that innovation does not affect the element technologies that have been outsourced previously to suppliers. 
Since short-term profits can be enhanced through outsourcing, while long-term profits benefit from coordinated innovation in multiple elements simultaneously ("long jumps"), inter-firm R\&D collaborations have been considered as an intermediate and flexible institutional solution to the coordination problem in complex systems. This insight has led to a rich literature on the rationales for inter-firm collaboration in $\mathrm{R} \& \mathrm{D}$ and the factors causing success and failures of such collaborative agreements. However, apart from institutional solutions to the coordination problem in complex systems, one is also led to the question what type of technical devices exist that overcome coordination requirements. Over the past decade, increasing attention has been paid to the virtues of "decomposability" and "modularity" in system architectures, which allow for parallel and uncoordinated innovation in different elements of a complex system. This is made possible through the use of interface standards that mediate the epistatic effects between the different elements in a complex technological system (Baldwin and Clark, 2000; Langlois and Robertson, 1992; Schilling, 2000).

\subsection{Decomposability versus modularity}

The concepts of decomposability and modularity can be addressed analytically within the NK-framework as presented above. It is argued in the following that one should carefully distinguish between decomposability and modularity of system architectures. Decomposability can be readily addressed in the original Kauffman-type NK-model, while modularity required a generalised NK-model to account for the special nature of interface standards.

In an earlier article (Frenken et al., 1999) it has been shown how one can model decomposable systems in the original NK-framework where the number of elements equal the number of functions. Decomposable systems can be defined as systems containing subsets of elements for which holds that all epistatic relations are contained within these subsets and that no epistatic relations exist between subsystems (Simon, 1969). An example of a decomposable architecture is given in Fig. 5. In this example, epistatic relations are present between $n=1$ and 2 and between $n=3$ and 4 .

A mutation in the first subsystem $(n=1,2)$ will not affect the fitness values of the elements contained in the second subsystem $(n=3,4)$ and vice versa. The decomposability of the system thus allows a firm to outsource one subsystem while controlling innovative activity in the second subsystem. Moreover, decomposable systems allow a system integrator to combine different designs for each subsystem in different product variants meeting the demands of different users, while profiting from economies of scope (Frenken, 2006).

Importantly, the decomposability of a system cannot be derived from its complexity as indicated by $K$. Depending on the structure of epistatic links in an architecture, systems with the same $K$-value can have different degrees of decomposability. Using the concept of cover, the decomposability of a complex systems can be indicated by the system's cover size (Page, 1996). The cover of a complex system is the size of the largest subsystem into which a system can be decomposed

$\begin{array}{ccccc} & \mathrm{n}=1 & \mathrm{n}=2 & \mathrm{n}=3 & \mathrm{n}=4 \\ \mathrm{w}_{1} & \mathrm{x} & \mathrm{x} & - & - \\ \mathrm{w}_{2} & \mathrm{x} & \mathrm{x} & - & - \\ \mathrm{w}_{3} & - & - & \mathrm{x} & \mathrm{x} \\ \mathrm{w}_{4} & - & - & \mathrm{x} & \mathrm{x}\end{array}$

Fig. 5. Example of a decomposable architecture of $N=4$-system with $K=1$ (Frenken et al., 1999). 
(in the example of Fig. 5, both subsystems have size two, so cover size would equal two). Using this measure, one can show that even NK-systems with a small $K$-value generally have a cover size as large as $N$, which means that most NK-systems are completely non-decomposable (Frenken et al., 1999). This outcome can be understood as resulting from the property of NK-systems that all elements are by definition epistatically affected by $K$ other elements, while each element affects only on average $K$ other elements.

The notion of decomposability as explained above, however, is only of limited use in the context of discussing outsourcing for two reasons. First, it is questionable whether there exist examples of technological systems that can be described as completely decomposable (Simon, 1969). Products (like personal computers and stereo sets) are complex wholes in the sense that the subsystems are physically interconnected through interfaces. Though different elements can often be produced independently, the product functions as a single system through the use of technical interface standards that interconnect the different modules. Second, even if one can speak of decomposability in the above sense, there would be no longer a role for a system integrator to assemble the product as suppliers can by-pass an integrator through direct offering of subsystems to users that combine the subsystems in such a way that the collective properties are produced. The question becomes how one can conceptualise non-decomposable yet modular architectures. This question has been highlighted by several authors writing on modularity and interface standardisation (Baldwin and Clark, 2000; Langlois and Robertson, 1992; Schilling, 2000), but has not yet been addressed within the context of the NK-model.

The 'power of modularity' in terms of facilitating innovation can be directly related to pleiotropy of a system's architecture. The lower the pleiotropy of an element in an architecture, the larger the likelihood of success of a mutation as increases in one function are less likely to be offset by decreases in fitness of other functions (Altenberg, 1995). Put another way, the higher the pleiotropy of an element, the more trade-offs between the fitness values of different functions exist, the higher the probability that the increases in fitness of some functions due to a mutation are offset by reductions in fitness of other functions. ${ }^{3}$

An example of a modular system has in fact already been given in Fig. 4 above. In that example, the second element can be considered as a interface standard that mediates the interdependencies between the other elements. The use of standards in complex production systems thus allows a firm to render its production system modular in the sense that a mutation in one element can be implemented without negatively affecting the functioning of other elements in the system. The example in Fig. 4 shows that systems can be completely modular without being decomposable: each function can be improved by mutation in a single element without epistatic effects on other functions.

The possibility of constructing a modular system is not restricted by the size of the system $N$. Below in Fig. 6, the logic of modularisation is repeated for a system of size $N=7$. In this system, the first and second elements function as standards affecting several functions (pleiotropy $>1$ ) mediating the interaction between all other elements, each of which affect only one function (pleiotropy =1). Note that the example in Fig. 6 is not a decomposable system as the system cannot be split up in two subsystems containing all epistatic relations. The set of functions affected by the first and second elements are overlapping (both affect the third function). ${ }^{4}$ A well-elaborated

\footnotetext{
${ }^{3}$ The higher the pleiotropy of an element, the closer the expected net result of a mutation in this element on system fitness $W$ will be to the expected value of a random draw 0.5 .

4 One can also model multi-layered modular systems, in which modules contain modules (Frenken and Mendritzki, 2005).
} 


\begin{tabular}{cccccccc} 
& $n=1$ & $n=2$ & $n=3$ & $n=4$ & $n=5$ & $n=6$ & $n=7$ \\
\hline$f=1$ & $x$ & - & $x$ & - & - & - & - \\
$\mathrm{f}=2$ & $x$ & - & - & $x$ & - & - & - \\
$\mathrm{f}=3$ & $\mathrm{x}$ & $\mathrm{x}$ & - & - & $\mathrm{x}$ & - & - \\
$\mathrm{f}=4$ & - & $\mathrm{x}$ & - & - & - & $\mathrm{x}$ & - \\
$\mathrm{f}=5$ & - & $\mathrm{x}$ & - & - & - & - & $\mathrm{x}$
\end{tabular}

Fig. 6. Example of a non-decomposable system with five modular elements $(N=7, F=5)$.

empirical example of a modular architecture as expressed in a matrix of epistatic interdependencies can be found in Baldwin and Clark (2000). Their main case in point is IBM 360, which was explicitly designed in a way to obtain maximum modularity.

What is important to note is that the high-pleiotropy elements mediating the interactions of other elements need not be considered as pure technical standards in terms of interface standards in the conventional sense. These high-pleiotropy elements can also refer to particular "core components" in the system, which happen to affect many functions. For example, the type of engine used in a car is expected to affect many functions of a car in conjunction with other elements that only affect one function. As such, the choice of engine resembles the choice for a technical standard in the sense that it is the yardstick for the choice of other elements. Once a choice of allele of high-pleiotropy elements is made, the system can be optimised by local search in low-pleiotropy elements. Hereafter, mutation in a high-pleiotropy element is no longer likely be successful since many functions in the system will be simultaneously be affected, the net result in system fitness generally being a loss (Altenberg, 1995, 1997; Frenken, 2006).

The existence of high-pleiotropy core elements explains why technologies are often standardised not only with regard to technical interface standards, but also with regard to core components. "Dominant designs" are often expressed in terms of the invariance of high-pleiotropy elements (like the engine or material), while peripheral low-pleiotropy elements are mutated very often to offer specific variants to a specific user group (e.g. luxury versions, fashionable versions) (Clark, 1985; Tushman and Murmann, 1998; Murmann and Frenken, 2002).

Modular architectures thus allow a system integrator to outsource low-pleiotropy elements without frustrating future innovation by suppliers. In contrast, high-pleiotropy elements such as technical standards or core components are less likely to be outsourced as mutations in these elements require coordination. Importantly, although modular architectures ex ante coordinate the innovative activities of suppliers at the modular level, they limit the scope for innovation of system integrators. The choice of standards, once established, is expected to be inert as a change in standards would undo the advancements made at the modular level. In the generalised NK-model, this logic of inertia can be explained by the lower probability of raising fitness by mutating an element with higher pleiotropy, since high pleiotropy means that increases in fitness of one set of functions is likely to be offset by decreases in fitness in another set of functions. A good empirical example of the rigidity of standards is provided by a recent study on innovation and competition in the LAN industry (Brusoni and Fontana, 2005). They found that modularity offered advantages in terms of modular changes, yet also generates a 'tunnel effect' that prevents firms from developing radically new products. Incumbents firms held on the standards that were based on a shared bus, while it were new entrants who developed a radically different technological design based on a switched bus:

"Since the early days of the industry, LAN functioning had been based on the principle that the communication channel had to be shared among the users who wanted access to 
it. Some manufacturers thought that implementing a switched bus in equipment such as hubs, which had always been quite simple from the technological viewpoint, might be difficult and encounter user resistance. Moreover, most of the available chipsets for hubs had been designed to work with LAN standards such as Ethernet which, because of the way they functioned, naturally supported a shared bus design. Implementing these chip sets in a switched bus design would have made the equipment less efficient. Because of these concerns, manufacturers' differed in their choice of solution. In particular, the 'switched' solution was adopted first by start-ups, namely Kalpana Inc. and Synernetics Inc. while the incumbents initially opted for the more conservative solution" (Brusoni and Fontana, 2005: 12-13).

An interesting aspect of their case study lies in the fact that the newly developed technological design was not based on new technological competencies as it was based on a recombination of existing and well-known principles within the telecommunication industry (Brusoni and Fontana, 2005). Thus, the failure of established firms to develop the radical innovation cannot be attributed to a supposed competence-destroying nature of radical innovation. Rather, it is suggested that their failure relates to their sunk investments, both financial and cognitive, in the older technology, which had evolved in a modular fashion around a rigid set of interface standards. Other case study examples of such a 'tunnel effect' of a modular innovation strategy, preventing radical solutions to be developed, can be found in Chesbrough and Kusunoki (2001) and Garud and Munir (2003).

\section{An indicator of modularity}

In the previous section an analytical definition of modularity of complex systems has been derived using the generalised NK-model. The concept of modularity as presented above allows one to supplement product life-cycle models with regard to the co-evolution of technical change and vertical organisation of production. Once it is possible to indicate the degree of modularity of different products, alternative patterns of vertical organisation can be related to differences in modularity.

A particular interesting area to address the concept of modularity concerns the product life-cycle studies of the evolution of different industries (Abernathy and Utterback, 1978; Klepper, 1997; Klepper and Miller, 1995; Tushman and Murmann, 1998). As it has been noted before (Klepper, 1997), the issue of vertical integration is very scarcely addressed hitherto within the context of the product life-cycle framework, which traditionally focused on changes in the horizontal organisation of industries over time (i.e. increasing concentration). Theoretically, the growing size of the market over the product life-cycle suggests that, as 'the division-of-labour is limited by the extent of the market', vertical disintegration would increase over the product life-cycle. However, empirical evidence on vertical integration in the product life-cycle literature is not conclusive: both examples of increasing vertical integration and increasing vertical disintegration have been reported (Klepper, 1997). To date, there have been no systematic attempts to explain these differences.

One way to approach the different histories of vertical integration during the product lifecycles is to analyse the modularity of different dominant designs in different industries. There is to date no theoretical reason to expect the degree of modularity of dominant designs to be roughly similar. Both "integrated" dominant designs and modular dominant designs can emerge over time. However, the effects of integrated or modular designs on the vertical organisation of production are expected to be very different. The degree of modularity of different product architectures can 
be used as an explanatory variable of the degree of vertical disintegration within a conventional product life-cycle framework.

An empirical study of modularity and vertical disintegration would require a measure of modularity of product architectures. Using the generalised NK-model above one can relate modularity to the pleiotropy values of elements in the matrix describing a system's architecture. Maximisation of modularity of non-decomposable systems is achieved by minimisation of the pleiotropy of elements under the restriction that the system is non-decomposable (i.e., no overlapping subsets of elements that exclusively affect subsets of functions). This means that at least some elements will have a pleiotropy higher than one in order to integrate the elements with pleiotropy equal to one. To maximise to number of elements with pleiotropy one, while integrating these through the use of standards, a single standard of maximum pleiotropy $F$ already suffices, yet the use of several standards with lower pleiotropy is also possible. The system pleiotropy is therefore to be expressed not by the sum of pleiotropy values but by the product of pleiotropy values: a more skewed distribution of pleiotropy values implies that interdependencies center around few standards allowing other elements to contribute to the modularity of the system.

Let the pleiotropy of each element be denoted by $P_{n}$, which is a value between 1 and $F$. The system pleiotropy can then be expressed by the logarithm with base $F$ of the product of the pleiotropy values of each element, yielding a system pleiotropy measure between 1 and $N$ :

$$
P={ }^{F} \log \left(\prod_{n=1}^{N} P_{n}\right) \quad(F>1)
$$

The minimum possible system pleiotropy, which is the case when all elements have minimum pleiotropy except for one element with maximum pleiotropy $F$, equals:

$$
P_{\min }={ }^{F} \log (F)=1
$$

The maximum possible system pleiotropy, which is the case when all elements have maximum pleiotropy $F$, equals:

$$
P_{\max }={ }^{F} \log \left(F^{N}\right)=N
$$

System modularity can now be expressed as an inverse function of system pleiotropy in the following way:

$$
M=\frac{\text { max system pleiotropy }- \text { observed system pleiotropy }}{\text { max system pleiotropy }}
$$

which gives:

$$
M=\frac{{ }^{F} \log \left(F^{N}\right)-{ }^{F} \log \left(\prod_{n=1}^{N} P_{n}\right)}{F \log \left(F^{N}\right)} \quad(F>1)
$$

which can be rewritten as:

$$
M=1-\frac{P}{N}
$$

The minimum possible system modularity $M$ is independent of system size $N$ :

$$
M_{\min }=1-\frac{N}{N}=0
$$


and the maximum possible system modularity is as a function of $N$ :

$$
M_{\max }=1-\frac{1}{N}
$$

The maximum possible system modularity has the property of approaching 1 when $N$ approaches infinity. Put differently, non-decomposable systems with infinite size will have a maximum modularity when all elements have pleiotropy one except for one element having pleiotropy $F$ functioning as a technical standard. A second property of the modularity indicator holds that systems with different sizes $N$ can still be compared on a scale between 0 and 1 . This allows one to compare empirically products of different sizes in terms of their modularity.

The modularity indicator can be used to indicate the percentage of elements that is expected to be outsourced on technological grounds (i.e., not taken into account differences in transaction costs and production costs). In the case of maximum modularity all but one element can be outsourced as expressed by $1-(1 / N)$. In the case of minimum modularity, no single element is expected to be outsourced as expressed by 0 . For all other architectures, the modularity indicator acts as an estimation of the technical scope for outsourcing.

To give some examples: in Fig. 6 there are five elements with pleiotropy one and two elements with pleiotropy three. System pleiotropy adds up to $P={ }^{5} \log (9)=1.365$ and system modularity to $M=1-(1.365 / 7)=0.805$. Modularity can still be enhanced by replacing the two interface standards by one with maximum pleiotropy. System pleiotropy would then add up to $P={ }^{5} \log (5)=1$ and system modularity to $M=1-(1 / 6)=0.833$, which is the maximum value for $N=6$.

Building modularity indicators on the basis of pleiotropy values is a straightforward way to characterise the architecture of complex systems. The matrix of elements and function from which pleiotropy values are derived can be constructed for many technologies (and sometimes have been constructed by designers for their own purposes, e.g., Alexander, 1964). The laptop example provided by Baldwin and Clark (2000, p. 74) can serve as an exemplar of how to draw such a matrix, from which further analysis can proceed. The matrix they present clearly shows five interface standards (columns 1-5), four modules (columns 6-33) and an integrating system (columns 34-39). The modules can be distinguished from the interface standards and integrating system, because the functioning of modules is solely dependent on the choices within each module and the choice of interface standard. This is reflected in the high pleiotropy of the five interface standard (17.6 on average) compared to the much lower pleiotorpy of the elements belonging to the modules (about five). As long as interface standards are chosen in the stage preceding the design of the modules (what they call stage 1), and remain in tact afterwards, the modules can be developed independently and in parallel (what they call stage 2), before being reintegrated in the integrating system (what they call stage 3).

\section{Conclusion}

Technical standards in complex systems do not produce particular functionalities but solely mediate the interdependencies between other elements. The original NK-model by Kauffman in which the number of elements equals the number of functionalities, falls short of describing standards. This paper introduced the generalised NK-model by Altenberg in the context of complex technological systems to account for the special nature of interface standards.

The mediation of epistasis among elements by standards facilitates vertical disintegration in two ways. Static transactions costs are reduced as the required properties of outsourced elements are solely dependent on the nature of the interface standard and can be codified more easily in 
contracts. Dynamic transaction costs are reduced too, because standards allow for independent innovative activity by suppliers as mutations in modules do not affect the fitness of other modules. Both suppliers and system integrators can continue to improve product quality of the elements they produce. This enduring division-of-labour allows individual parties to specialise both in production competences and innovation competences within a vertical chain of production of a complex system.

It is hoped that the NK approach to modularity and decomposability presented here, will be integrated in future modelling exercises. An immediate question that comes to mind is whether search heuristics perform differently in optimising modular systems compared to optimising standard NK-systems (Frenken et al., 1999). Furthermore, one expects decentralised search (Kauffman and Macready, 1995; Rivkin and Siggelkow, 2003; Siggelkow and Levinthal, 2003) to perform much better in optimising modular systems compared to optimising standard NK-systems. Such questions can be researched systematically by applying the proposed definition of modularity.

Finally, using the generalised NK-model, it has been argued here that one should carefully distinguish between decomposability and modularity. In decomposable systems innovation within a subsystem is not hampered by other subsystems, since interdependencies are absent between subsystems. In modular, yet non-decomposable systems, innovation is possible in modules to the extent that the innovation meets the requirements of interface standards that integrate the modules into a system. Modularity thus provides a way to implement division-of-labour, and possibly outsourcing, even in the absence of full decomposability. A measure of modularity has been proposed that can be used in empirical studies on product evolution and vertical organisation. The higher the modularity of a system as defined above, the lower the degree of vertical disintegration, ceteris paribus. This proposition can be tested by analysing the pleiotropy of architectures of different products and of single products over time.

\section{References}

Abernathy, W.J., Utterback, J., 1978. Patterns of industrial innovation. Technology Review 50, 41-47.

Alexander, C., 1964. Notes on the Synthesis of Form. Harvard University Press, Cambridge, MA.

Altenberg, L., 1995. Genome growth and the evolution of the genotype-phenotype map. In: Banzhaf, W., Eckman, F.H. (Eds.), Evolution and Biocomputation. Springer-Verlag, Berlin \& Heidelberg, pp. 205-259.

Altenberg, L., 1997. NK fitness landscapes. In: Back, T., Fogel, D., Michalewicz, Z. (Eds.), The Handbook of Evolutionary Computation. Oxford University Press, Oxford.

Arthur, W.B., 1989. Competing technologies, increasing returns, and lock-in by historical events. Economic Journal 99 , $116-131$.

Auerswald, P., Kauffman, S., Lobo, J., Shell, K., 2000. The production recipes approach to modeling technological innovation: an application to learning by doing. Journal of Economic Dynamics \& Control 24 (3), 389-450.

Baldwin, C.Y., Clark, K.B., 2000. Design Rules. The Power of Modularity, vol. 1. MIT Press, Cambridge, MA.

Bradshaw, G., 1992. The airplane and the logic of invention. Minnesota Studies in the Philosophy of Science 15, 239-250.

Brusoni, S., Fontana, R., 2005. Modularity as an entry strategy: the invasion of new niches in the LAN equipment industry. CESPRI Working Papers, 171, Bocconi University, Milan.

Buenstorf, G., 2005. Sequential production, modularity and technological change. Structural Change and Economics Dynamics 16 (2), 221-241.

Chesbrough, H., Kusunoki, K., 2001. The modularity trap: innovation, technology phase shifts, and the resulting limits of virtual organizations. In: Nonaka, I., Teece, D. (Eds.), Managing Industrial Knowledge: Creation, Transfer and Utilization. Sage, Thousand Oaks, CA, pp. 2002-2230.

Clark, K.B., 1985. The interaction of design hierarchies and market concepts in technological evolution. Research Policy $14,235-251$.

David, P.A., 1985. The economics of QWERTY. American Economic Review (Papers \& Proceedings) 75, $332-337$.

Dennett, D., 1995. Darwin's Dangerous Idea. Penguin, paperback edition, 1996. London.

Fleming, L., 2001. Recombinant uncertainty in technological search. Management Science 47 (1), 117-132. 
Fleming, L., Sorenson, O., 2001. Technology as a complex adaptive system: evidence from patent data. Research Policy 30 (7), 1019-1039.

Foss, N.J., 1993. Theories of the firm: contractual and competence perspectives. Journal of Evolutionary Economics 3 , $127-144$.

Frenken, K., 2006. Innovation, Evolution and Complexity Theory. Edward Elgar, Northampton.

Frenken, K., Marengo, L., Valente, M., 1999. Interdependencies, near-decomposability and adaptation. In: Brenner, T. (Ed.), Computational Techniques for Modelling Learning in Economics. Kluwer, Boston, pp. 209-244.

Frenken, K., Mendritzki, S., 2005. Global optimisation of modular systems, Manuscript, 19 July, Utrecht University.

Frenken, K., Nuvolari, A., 2004. The early development of the steam engine: an evolutionary interpretation using complexity theory. Industrial and Corporate Change 13 (2), 419-450.

Garud, R., Munir, K., 2003. Socio-technical dynamics underlying radical innovation: the case of Polaroid's Sx-70 camera, Paper presented at the SPRU conference What do we know about innovation? University of Sussex, 13-15 November.

Henderson, R., Clark, K., 1990. Architectural innovation. Administrative Science Quarterly 35, 9-30.

Kauffman, S., Lobo, J., Macready, W.G., 2000. Optimal search on a technology landscape. Journal of Economic Behavior \& Organization 43 (2), 141-166.

Kauffman, S.A., 1993. The Origins of Order. Self-organization and Selection in Evolution. Oxford University Press, New York \& Oxford.

Kauffman, S.A., Macready, W.J., 1995. Technological evolution and adaptive organizations. Complexity 1, $26-43$.

Klepper, S., 1997. Industry life-cycles. Industrial and Corporate Change 6, 145-182.

Klepper, S., Miller, J.H., 1995. Entry, exit, and shakeouts in the United States in new manufactured products. International Journal of Industrial Organization 13 (4), 567-591.

Langlois, R.N., 1992. Transaction-cost economics in real time. Industrial and Corporate Change 1 (1), 99-127.

Langlois, R.N., 2001. The vanishing hand: the changing dynamics of industrial capitalism, Paper presented at the workshop Reappraising Production Theory: Concepts, Cases and Models, Max Planck Institute, Jena, November 29-December 1.

Langlois, R.N., Foss, N.J., 1999. Capabilities and governance: the rebirth of production in the theory of economic organization. Kyklos 52 (2), 201-218.

Langlois, R.N., Robertson, P.L., 1992. Networks and innovation in a modular system - lessons from the microcomputer and stereo component industries. Research Policy 21 (4), 297-313.

Levinthal, D.A., 1997. Adaptation on rugged landscapes. Management Science 43 (7), 934-950.

Levinthal, D.A., Warglien, M., 1999. Landscape design: designing for local action in complex worlds. Organization Science 10 (3), 342-357.

Mahnke, V., 2001. The process of vertical disintegration: an evolutionary perspective on outsourcing. Journal of Management and Governance 5, 353-379.

Marengo, L., Dosi, G., Legrenzi, P., Pasquali, C., 2000. The structure of problem-solving knowledge and the structure of organizations. Industrial and Corporate Change 9, 757-788.

McKelvey, B., 1999. Avoiding complexity catastrophe in coevolutionary pockets: strategies for rugged landscapes. Organization Science 10 (3), 294-321.

Murmann, P., Frenken, K., 2002. Towards a systematic framework for research on dominant designs, technological innovations, and industrial change. Papers on Economics and Evolution, 0212, Max Planck Institute, Jena.

Page, S.E., 1996. Two measures of difficulty. Economic Theory 8, 321-346.

Prahalad, C., Hamel, G., 1990. The core competence of the organization. Harvard Business Review 68, 79-91.

Rivkin, J.W., 2000. Imitation of complex strategies. Management Science 46 (6), 824-844.

Rivkin, J.W., 2001. Reproducing knowledge: replication without imitation at moderate complexity. Organization Science 12 (3), 274-293.

Rivkin, J.W., Siggelkow, N., 2003. Balancing search and stability: interdependencies among elements of organizational design. Management Science 49, 290-311.

Rosenberg, N., 1969. The direction of technical change: inducement mechanisms and focussing devices. Economic Development and Cultural Change 18, 1-24.

Saviotti, P.P., 1996. Technological Evolution, Variety and the Economy. Edward Elgar, Cheltenham \& Brookfield.

Saviotti, P.P., Metcalfe, J.S., 1984. A theoretical approach to the construction of technological output indicators. Research Policy 13, 141-151.

Schilling, M.A., 2000. Toward a general modular systems theory and its application to interfirm product modularity. Academy of Management Review 25 (2), 312-334.

Siggelkow, N., Levinthal, D.A., 2003. Temporarily divide to conquer: centralized, decentralized, and reintegrated organizational approaches to exploration and adaptation. Organization Science 14 (6), 650-669. 
Simon, H.A., 1969. The Sciences of the Artificial. MIT Press, Cambridge, MA \& London.

Teece, D.J., 1976. Vertical Integration and Vertical Divestiture in the U.S. Oil Industry. Institute for Energy Studies, Stanford, CA.

Tushman, M.L., Murmann, J.P., 1998. Dominant designs, technology cycles, and organizational outcomes. Research in Organizational Behavior 20, 231-266.

Valente, M., 2000. Evolutionary economics and computer simulations. A model for the evolution of markets. Aalborg University. Ph.D. Thesis, http://www.busines.auc.dk/ mv.

Williamson, O.E., 1999. Strategy research: governance and competence perspectives. Strategic Management Journal 20 (12), 1087-1108.

Williamson, O.E., 1975. Market and Hierarchies. Free Press, New York.

Williamson, O.E., 1979. Transaction-cost economics: the governance of contractual relations. Journal of Law and Economics 22, 233-261.

Williamson, O.E., 1985. The Economic Institutions of Capitalism. Free Press, New York.

Winter, S.G., 1988. On coarse, competence, and the corporation. Journal of Law Economics \& Organization 4, $163-180$. 